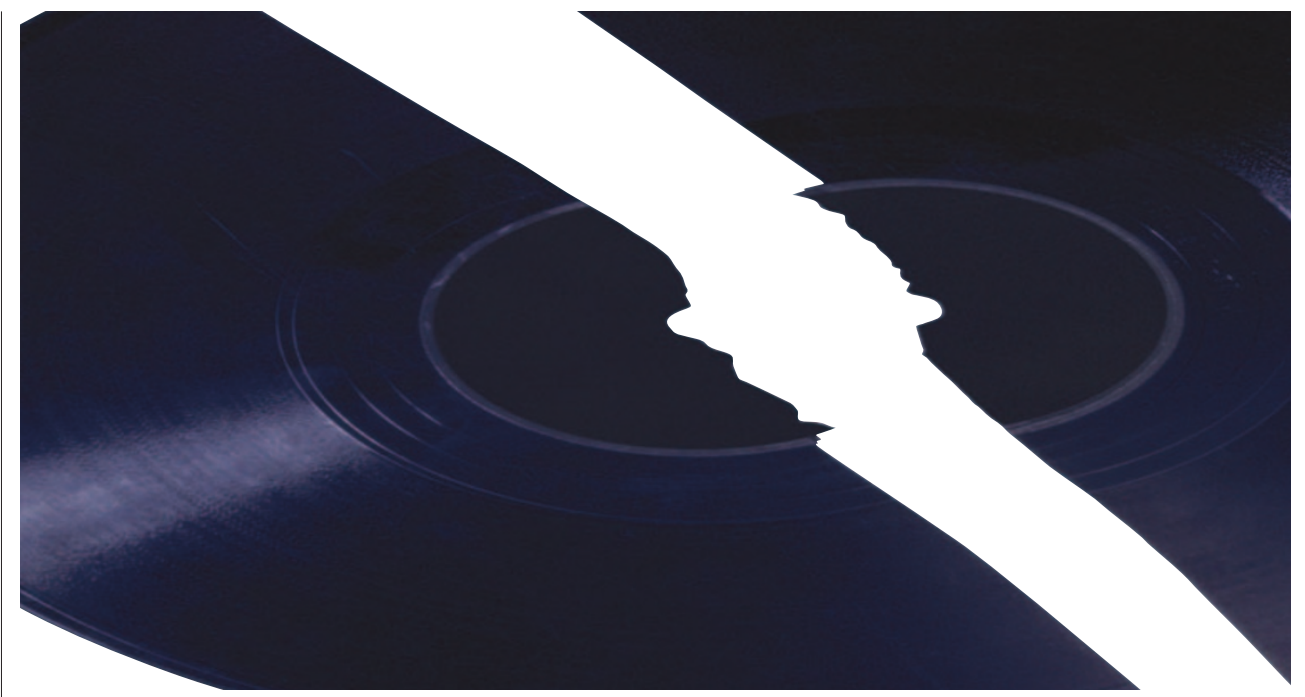

DVIROLOGY

\title{
Promoting silence
}

Herpesviruses are characterized by their ability to establish latent infections from which the virus can reactivate and cause recurrent disease. The transition from productive (lytic) to latent infection in herpes simplex virus (HSV) is associated with marked shutdown of the herpesvirus genome - during lytic infection more than 80 gene products are expressed, whereas during latent infection only the latency-associated transcript gene ( $L A T)$ is highly expressed. Now, David Knipe and colleagues argue that $L A T$ represses HSV gene expression by promoting the assembly of heterochromatin on 
viral lytic-gene promoters.

The authors used chromatin immunoprecipitation assays to study the assembly of chromatin on HSV lytic genes during infection of murine trigeminal ganglia. Initially, during lytic infection, low levels of histone $\mathrm{H} 3$ were associated with viral DNA, which is consistent with findings that the viral genome is relatively nucleosome-free during productive infection. But as latent infection was established, the genome became increasingly chromatinized. Consistently, during latent infection, lytic-gene promoters showed a higher level of association with heterochromatin (marked by histone $\mathrm{H} 3$ methylated at lysine 9) compared with euchromatin (marked by histone $\mathrm{H} 3$ methylated at lysine 4). Using $L A T^{-}$HSV, Wang et al. showed that expression of $L A T$ significantly increased the amount of heterochromatin and decreased the amount of euchromatin associated with most lytic-gene promoters. This suggests that LAT facilitates shutdown of the HSV genome by promoting the assembly of inaccessible chromatin on viral DNA.

The authors acknowledge that this accumulation of heterochro- matin on the lytic-gene promoters might be an indirect process that takes place on loci silenced by a mechanism unrelated to chromatin. Nevertheless, the proposal that $L A T$ directly affects viral chromatin structure is appealing. Doublestranded RNA has been shown to target the formation of heterochromatin through the RNA interference machinery. As yet, LAT has not been shown to encode protein products, and therefore the authors contend that LAT RNA might form small interfering RNAs that could induce heterochromatin formation on lytic-gene promoters.

The establishment of a quiescent form of HSV allows the virus to remain hidden from the host immune system and prevents the induction of apoptosis in infected cells. Understanding the mechanism of latency could therefore prove crucial in the development of effective therapies.

Shannon Amoils

ORIGINAL RESEARCH PAPER Wang, Q-Y. et al. Herpesviral latency-associated transcript gene promotes assembly of heterochromatin on viral lytic-gene promoters in latent infection. Proc. Natl Acad. Sci. USA 102, 16055-16059 (2005) WEB SITE

David Knipe's laboratory:

http://knipelab.med.harvard.edu 